\title{
Norspermidine changes the basic structure of $S$. mutans biofilm
}

\author{
MEIZHEN OU and JUNQI LING \\ Department of Operative Dentistry and Endodontics, Guanghua School of Stomatology, Provincial Key Laboratory of Stomatology, \\ Sun Yat-sen University, Guangzhou, Guangdong 510055, P.R. China
}

Received October 15, 2015; Accepted October 26, 2016

DOI: $10.3892 / \mathrm{mmr} .2016 .5979$

\begin{abstract}
The factors regulating the assembly of the three-dimensional structure of Streptococcus mutans biofilms remain obscure. Polyamines are essential in biofilm formation of certain bacteria. Norspermidine, an unusual polyamine, has been a controversial polyamine that can lead to biofilm disassembly. However, the role of norspermidine in $S$. mutans biofilms remains unknown. Therefore, the present study investigated the impact of norspermidine on $S$. mutans biofilms. The different architectures of the biofilms in norspermidine and control groups indicated that the basic units, bacteria-exopolysaccharide units (BEUs), represent the exopolysaccharide (EPS) and bacterial assembly pattern in $S$. mutans biofilms. In addition, norspermidine inhibited $S$. mutans biofilm formation and changed the basic composition of the biofilm, which led to an unusual EPS architecture. Therefore, $5 \mathrm{mM}$ norspermidine inhibited biofilm formation both by decreasing the rate of cell viability and changing the biofilm structure. Gene-expression microarray analysis indicated that the formation of an irregular architecture in the norspermidine group was potentially attributable to the downregulation of elements of the quorum-sensing system (by 2.7-15-fold). The present study suggested that the BEUs are a basic structure of $S$. mutans biofilm and its assembly is regulated majorly by the quorum-sensing system. Norspermidine can lead to structure change in BEUs by influencing $S$. mutans quorum-sensing system.
\end{abstract}

\section{Introduction}

Biofilms are multicellular communities of bacteria encased in an extracellular matrix of exopolysaccharides (EPS), proteins and, occasionally, DNA $(1,2)$. EPS are thought to maintain

Correspondence to: Professor Junqi Ling, Department of Operative Dentistry and Endodontics, Guanghua School of Stomatology, Provincial Key Laboratory of Stomatology, Sun Yat-sen University, 56 Ling Yuan Xi Road, Guangzhou, Guangdong 510055, P.R. China E-mail: lingjq@mail.sysu.edu.cn

Key words: Streptococcus mutans, biofilm, norspermidine, quorum-sensing system, glucosyltransferases, glucan-binding proteins, spaP the architecture of the biofilm and to protect the bacteria by harboring them within the biofilm. The composition and structure of the EPS component may vary according to the microbial constituents, which are affected by local environmental conditions; for example, by shear forces in fluid environments, and can change over time (3-5). EPS are a key component of the matrix of cariogenic oral biofilms and are known virulence factors in the pathogenesis of dental caries (6). EPS create complex three-dimensional structures and compartmentalized acidic microenvironments within a biofilm, which trigger the dominance of pathogenic Streptococcus mutans within a mixed-species system (7). Glucans constitute the majority of the EPS in S. mutans biofilms (8-10). S. mutans synthesizes glucans from dietary sucrose via glucosyltransferases (GTFs). These enzymes are virulence factors in the etiology and pathogenesis of dental caries (9). Glucans promote the adherence and accumulation of $S$. mutans on the tooth surface and serve an essential role in the development of caries-forming activity (6). The majority of previous research has focused on the bacterial aspects of these biofilms, whereas the assembly of the $S$. mutans EPS and the functional roles that these EPS serve in the microcosmic basic structures of these biofilms have received limited attention.

$S$. mutans lives on the tooth surface at a high cell density in dental plaque. In a process termed quorum sensing (QS), which was first discovered in the marine bacterium Vibrio fischeri, bacterial populations coordinate the behavior of the community. It is now apparent that QS systems exist in both Gram-positive and Gram-negative bacteria, and that they have evolved to improve access to complex nutrients or environmental niches or to collectively enhance the defense against other microorganisms or eukaryotic-host defense mechanisms (11-14). QS bacteria convey their presence to one another by releasing and responding to the accumulation of low-molecular-weight chemical signaling molecules called autoinducers. Gram-positive bacteria generally accomplish interspecies communication via processed oligopeptides and these signals are detected via two-component signal transduction systems. S. mutans produces both AI-2 and competence-stimulating peptides (CSPs), which belong to the AI-1 family, via the $\operatorname{luxS}$ and $\operatorname{com} C$ genes, respectively. The relationship between QS and $S$. mutans biofilm structure has been investigated and discussed previously. The ability of bacterial cells to communicate and behave collectively as a group most likely provides significant benefits for the colonization of hosts, defense against competitors, adaptation 
to varying physical conditions, cellular differentiation and species evolution (15).

Polyamines are linear, organic polycations that are fully protonated at physiological $\mathrm{pH}$ and are observed in most bacterial species. Due to their cationic nature, polyamines are able to interact with negatively charged molecules within the cell, including DNA, RNA, proteins and phospholipids, thereby affecting their structure and function (16). Polyamines are known to serve important roles in biofilm formation, although the exact mechanisms remains to be elucidated. Polyamines have been observed to affect proteins that are important for biofilm formation in Yersinia pestis and Escherichia coli (17-19), and may serve as intercellular signaling molecules in Proteus mirabilis (19). Norspermidine (Nspd), with one methyl less than spermidine, serves a negative role in numerous bacteria, with the exception of Vibrio. In addition, exogenous Nspd may directly dissolve EPS, leading to the detachment of Bacillus subtilis biofilms, although this hypothesis remains controversial (20). However, information is limited regarding the association between polyamines and biofilm structure, particularly the EPS, which comprise major structures in biofilms.

In the present study, the impact of Nspd on $S$. mutans biofilm formation and biofilm three-dimensional structure were investigated in vitro. By analyzing the differentially expressed genes affected by Nspd, the present study proposed that the basic structure of the S. mutans biofilm appeared to be controlled by a QS system.

\section{Materials and methods}

Bacterial growth, agents and biofilm formation. Streptococcus mutans UA159 was obtained from Provincial Key Laboratory of Stomatology, Sun Yat-sen University (Guangzhou, China). The medium used included brain heart infusion broth (BHI; Becton Dickinson, Sparks, MD, USA). Nspd (Sigma-Aldrich, St. Louis, MO, USA) was used in the present study. The polyamine solutions were freshly prepared prior to each use.

Effective Nspd concentration on the biofilm formation of $S$. mutans and growth curve detection. Nspd at concentrations of 5, 2.5 and $1.25 \mathrm{mM}$, and $625,320,160,80$ and $5 \mu \mathrm{M}$ were added to the $S$. mutans suspensions in 96-well plates. The plates were subsequently incubated anaerobically at $37^{\circ} \mathrm{C}$ for $24 \mathrm{~h}$ without agitation. The biofilms that had formed in the BHI were washed twice and crystal violet $(\mathrm{CV})$ staining was performed to determine the biofilm biomass as the blank control.

Nspd was added to the $S$. mutans suspensions in tubes containing fresh liquid BHI to a final concentration of $5 \mathrm{mM}$ prior to incubation. The tubes were subsequently incubated anaerobically at $37^{\circ} \mathrm{C}$ for $12,24,36,48,60,72,84$ and $96 \mathrm{~h}$ without agitation. The number of viable bacteria in each of the cultures was determined in 10-fold serial dilutions with sterile water using the plate count methods. All experiments were performed at least in triplicate and cultures lacking polyamines were used as the blank control.

Effect of polyamines on biofilm disassembly and dynamic light scattering of EPS. Biofilms that had formed over $24 \mathrm{~h}$ without polyamines were washed twice to remove the media. Fresh BHI containing 1\% sucrose was added to the wells, and Nspd was added to a final concentration of $5 \mathrm{mM}$. Fresh BHI-sucrose was added to the control group. The plates were incubated at $37^{\circ} \mathrm{C}$ for $24 \mathrm{~h}$ under anaerobic conditions without agitation. Following this, the biofilms were washed twice and CV staining was performed to evaluate the biofilm biomass.

The dynamic light scattering measurements were performed according to methods reported previously, with some minor changes (20). Biofilms formed in BHI containing $1 \%(\mathrm{w} / \mathrm{v})$ sucrose for $24 \mathrm{~h}$ were harvested and washed twice to remove the supernatants. The biofilms were subsequently collected and resuspended in a $0.4 \mathrm{M} \mathrm{NaOH}$ solution. The cells were removed by centrifugation at $18,900 \times \mathrm{x}$ at $4^{\circ} \mathrm{C}$ for $10 \mathrm{~min}$, and the supernatants were mixed with cold isopropanol at a 5:1 ratio and incubated at $4^{\circ} \mathrm{C}$ overnight. The samples were centrifuged at 7,000 x $g$ at $4^{\circ} \mathrm{C}$ for $10 \mathrm{~min}$, and the pellets were harvested and subsequently lyophilized. The temperature was maintained at $25^{\circ} \mathrm{C}$. The dry, water-insoluble EPS samples were diluted in water and were gently sonicated at $200 \mathrm{~W}$ for $5 \mathrm{~min}$. The final EPS concentrations were adjusted to $30 \mathrm{mg} / \mathrm{ml}$. The light scattering value of each sample was measured prior to and after the addition of Nspd to a final concentration of $5 \mathrm{mM}$ or $10 \mathrm{mM}$. The dynamic light scattering measurements were obtained by focusing a vertically polarized light $(532 \mathrm{~nm})$ onto the sample and collecting the scattered light using a detector arranged at $90^{\circ}$. The data were collected at $30 \mathrm{sec}$ intervals for 10-30 min.

Stereomicroscopy. Biofilms that had formed in 24-well microtiter plates for $96 \mathrm{~h}$ in the presence of $5 \mathrm{mM}$ Nspd or control group were washed twice to remove the supernatant and were naturally air-dried at room temperature for $20 \mathrm{~min}$. The biofilms were observed and images were captured using a stereomicroscope at a magnification of x5 (Leica M205A; Leica Microsystems, Wetzlar, Germany).

Confocal laser-scanning microscopy (CLSM). The biofilms of each group were grown on glass-bottomed chamber slides for 12, 24, 36, 48, 60, 72, 84 and $96 \mathrm{~h}$. The biofilms were washed twice, stained using $200 \mu \mathrm{l}$ polysaccharide label Fluorescent Brightener 28 at 300 mg/l (Sigma-Aldrich) for $30 \mathrm{~min}$ and subsequently washed twice using PBS to remove the excess dye.

In the cell- and EPS-staining experiment, the samples were stained using the L-7012 LIVE/DEAD BacLight ${ }^{\mathrm{TM}}$ Bacterial Viability kit (Molecular Probes Inc., Eugene, OR, USA) according to the manufacturer's protocol after staining with Fluorescent Brightener 28. The live and dead cells in the biofilms were differentiated by staining using SYTO9 (green fluorescence) and propidium iodide (PI, red fluorescence). The samples were subsequently washed twice using PBS to remove the excess dyes.

Images of the stained specimens were captured using a Carl Zeiss LSM 780 confocal laser-scanning microscope (Carl Zeiss Microscopy, Jena, Germany) and analyzed using ZEN software (Zen 2012 light edition; Carl Zeiss MicroImaging, Inc., Thornwood, NY, USA). Three randomly selected areas of each biofilm were scanned. 
Microarray analysis of the gene expression

Samples. The biofilms in the control and Nspd groups were allowed to form for $48 \mathrm{~h}$ prior to harvesting by centrifugation and re-suspension in TRIzol reagent (Takara Bio Inc., Dalian, China). Each suspension was subsequently transferred to an RNase-free $1.5 \mathrm{ml}$ microcentrifuge tube. The quantity and quality of the RNA were evaluated using a NanoDrop ND-1000 instrument. The RNA integrity was assessed using standard denaturing agarose-gel electrophoresis.

DNA microarray. The Streptococcus mutans UA159 microarray is a broad array that represents well-known and predicted genes and transcripts. Coupled with the gene prediction capability of NCBI and the probe selection program of Agilent, this platform delivers data of better quality and has less redundant gene coverage.

RNA labeling and array hybridization. Sample labeling and array hybridization were performed according to the Agilent One-Color Microarray-Based Gene Expression Analysis protocol (Agilent Technology, Inc., Santa Clara, CA, USA). Briefly, the total RNA from each sample was linearly amplified and labeled using Cy3-UTP. The labeled cRNAs were purified using an RNeasy Mini kit (Qiagen GmbH, Hilden, Germany). The concentration and specific activity of the labeled cRNAs (pmol Cy3/ $\mu \mathrm{g}$ cRNA) were measured using a NanoDrop ND-1000 instrument. One microgram of each labeled cRNA was fragmented by adding $11 \mu \mathrm{l}$ of $10 \mathrm{X}$ blocking agent and $2.2 \mu \mathrm{l}$ of $25 \mathrm{X}$ fragmentation buffer, and heating the mixture to $60^{\circ} \mathrm{C}$ for $30 \mathrm{~min}$. Finally, $55 \mu \mathrm{l}$ of $2 \mathrm{X}$ GE hybridization buffer was added to dilute the labeled cRNA (GE Healthcare Life Sciences, Chalfont, UK). A total of $100 \mu$ l hybridization solution was dispensed into the gasket slide, which assembled on the gene expression microarray slide. The slides were incubated for $17 \mathrm{~h}$ at $65^{\circ} \mathrm{C}$ in an Agilent hybridization oven (Agilent Technology, Inc.). The hybridized arrays were washed, fixed and scanned using the Agilent DNA Microarray Scanner (part number, G2505C; Agilent Technology, Inc.).

Data analysis. Agilent Feature Extraction software (version 11.0.1.1; Agilent Technology, Inc.) was used to analyze the acquired array images. Quantile normalization and subsequent data processing were performed using the GeneSpring GX v11.5.1 software package (Agilent Technologies, Inc.). Following quantile normalization of the raw data, the genes expressed in at least two of the six samples were flagged as detected and were selected for further analysis. Genes that were significantly differentially expressed were identified through volcano plot filtering. Hierarchical clustering was performed using Agilent GeneSpring GX software (version 11.5.1). Pathway analysis was performed using the standard enrichment computation method.

Statistical analysis. All experiments were performed in triplicate and were repeated at least three times. The data were analyzed using SPSS (version 17.0 for Windows) software. Two-group comparisons were performed using Student's $t$-test. Significance was set as $\mathrm{P}<0.05$. The COMSTAT program was used to calculate the biomass, number and size (volume, diameter and thickness) of the microcolonies.

\section{Results}

Nspd effective concentration and growth curve in BHI. Reductions of $75.9 \%$ biofilm volume was observed in the Nspd group of $5 \mathrm{mM}$ concentration (Fig. 1A) $(\mathrm{P}<0.05)$. The growth curve revealed that S.mutans growth was inhibited during the early stages, then rebounded and reaching their growth peaks at $36 \mathrm{~h}$ (Fig. 1B).

Nspd had no direct effect on EPS nor caused any disassembly effect of $S$. mutans biofilm. In the disassembly experiment, Nspd was added to the cultures for $24 \mathrm{~h}$. Following this period, no significant differences in the optical density values of each group was observed $(\mathrm{P}>0.05)$. No collapse or vacuoles were observed in these groups using a Leica M205A stereomicroscope (Leica Microsystems, Wetzlar, Germany). As an independent approach to detect an interaction between Nspd and EPS, dynamic light-scattering experiments were performed. No direct effect of Nspd on the EPS was detected in this experiment.

Non-homogeneous general appearance of the biofilms in the experiment group was observed using stereomicroscopy. The biofilms produced in the control group were thick and firm, and formed intact blocks that were tightly attached to the surface of the plate. These biofilms had a smooth surface and uniform structure. The biofilms appeared to have white, smooth, confluent and translucent membranes (Fig. 2).

The biofilms of the Nspd group were very different compared with the control group. Their aggregates had a stellate shape and had no connection to each other. Rough, non-homogeneous and irregular structures were observed in these biofilms.

Diverse EPS architectures lead by Nspd observed using CLSM. The EPS granules in the control group appeared as globular structures and had similar sizes ranging from $\sim 5-25 \mu \mathrm{m}$ (Fig. 3). The uniform and regular EPS granules had a thickly dotted structure. The spaces within these EPS granules were uniform and ranged from $\sim 1-25 \mu \mathrm{m}$ in diameter. While in the experiment group, $S$. mutans formed heterogeneous EPS structures. Some of these EPS structures resembled fishnets and were wrinkled (Fig. 3, Nspd-a). Other structures were small and were connected to one another via chain-like structures (Fig. 3, Nspd-b). Larger structures that resembled pearls with a central hole or tube were also observed (Fig. 3, Nspd-c). This diverse EPS structure resulted in an irregular biofilm with varying thicknesses that appeared highly different from the biofilms produced by the other groups.

Three-dimensional distribution detail for basic structure units in S. mutans biofilms observed using CLSM. More detailed architectural differences were revealed in cells along with EPS stained observations (Fig. 4). Little structural differences were observed, however the number of dead cells increased between 24 and $96 \mathrm{~h}$ in the control group, while small aggregates expanded to a huge architecture in the experiment group. This Similarly sized and regularly shaped EPS granules were scattered equally throughout the biofilms in the control group. Within these structures was a 'space' lacking stained 

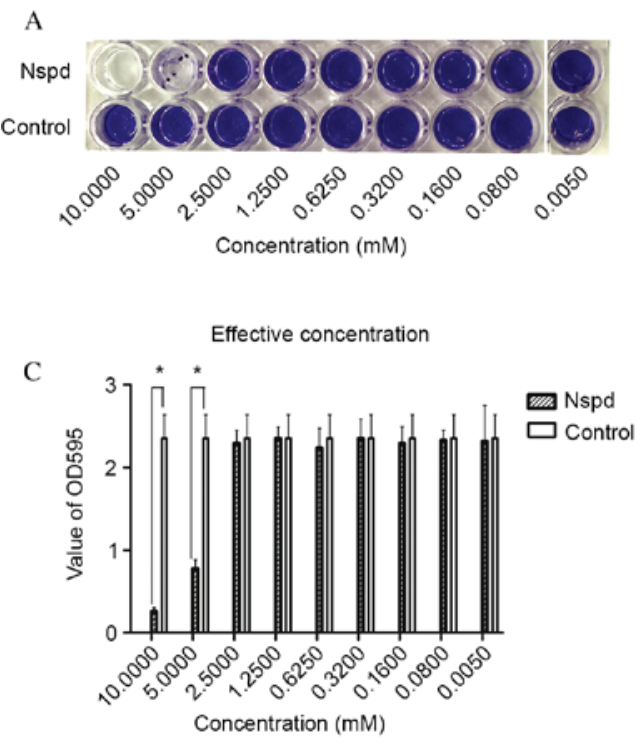

B

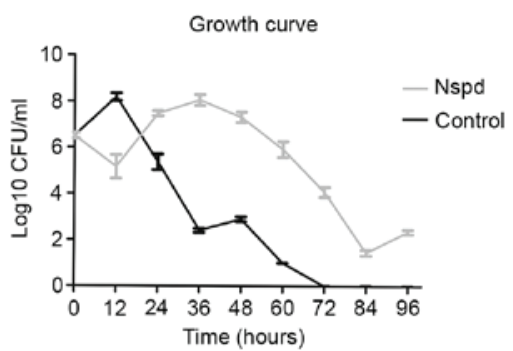

D

Dynamic light scattering measurements of WIG

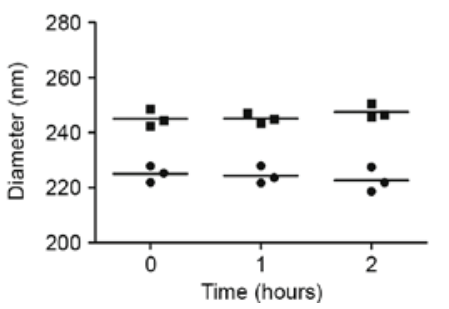

- $5 \mathrm{mM}$

- $10 \mathrm{mN}$

Figure 1. (A) Biofilms that formed following the addition of Nspd at $5 \mathrm{mM}$ and incubated for $24 \mathrm{~h}$, as visualized using crystal violet staining. (B) Growth curve of Streptococcus mutans. Nspd $(5 \mathrm{mM})$ was added to the suspension, which was incubated anaerobically at $37^{\circ} \mathrm{C}$ for $12,24,36,48,60,72,84$ and $96 \mathrm{~h}$ without agitation. The results were expressed as the mean \pm standard deviation of triplicate assays. (C) Biofilm production was evaluated using the OD595 values. The results are expressed as the mean \pm standard deviation of triplicate assays ( ${ }^{*} \mathrm{P}<0.05, \mathrm{n}=9$ paired Student's $t$-test). (D) Dynamic light scattering measurements. The diameters of the water-insoluble exopolysaccharide were determined following treatment with 5 or $10 \mathrm{mM}$ Nspd $(\mathrm{n}=3)$. Each measurement was repeated three times. Correlation analysis indicated no linear relationship between the diameter of the water-insoluble glucan and Nspd treatment (P>0.05). Nspd, norspermidine; CFU, colony forming units; OD, optical density; WIG, water-insoluble glucan.

bacteria, which made the ball-like structures appear to be hollow spheres (Fig. 5). The 'space' gradually narrowed from the adherent interface (bottom) to the biofilm surface. These types of 'hollow space' appeared to be completely filled with blue-stained EPS (Fig. 6). The cells surrounded the EPS formed three-dimensional ball- or stick-like structures. EPS was at the center of each ball-like bacterial cluster and exhibited an even gel-like brightness. This confirmed the exact basic structure assembly pattern of $S$. mutans biofilms. The basic structures of the biofilm included live and dead cells, which were organized into spheres or irregular-shaped balls (Fig. 5). These basic structures were uniform and well-distributed due to the size of each unit being $\sim 5-40 \mu \mathrm{m}$ in diameter (Figs. 4 and 6). The clusters were distributed in a regular pattern. The images demonstrated that the spherical structural units consisting of bacteria surrounding EPS were the basic units in the $S$. mutans biofilms (Fig. 6). The basic units were notably larger in the Nspd group (Figs. 4 and 7). The large EPS structures were irregular ball-like units with a thin bacterial layer on their surfaces, and there were holes in the center of the huge EPS clusters (Figs. 4 and 7). The course of the assembly of the EPS structures and the formation of the basic units in the Nspd group is shown (Figs. 4 and 7). During the first stage of $\sim 24 \mathrm{~h}$, a small number of bacterial clusters aggregated and successfully adhered to the surface of the chamber slide and began to secrete EPS toward the underside of the center of the clusters. With the cells continuing to secrete, the EPS with the units became more compact and abundant over time and formed a central hole.

Effect of Nspd on gene expression. To determine how Nspd leads to the formation of huge EPS clusters and basic structure, the present study examined the gene expression of cells in the biofilm compared with that of the control group. An overview

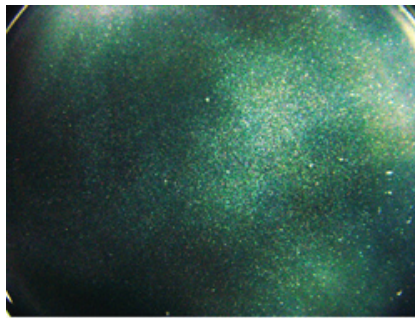

Control

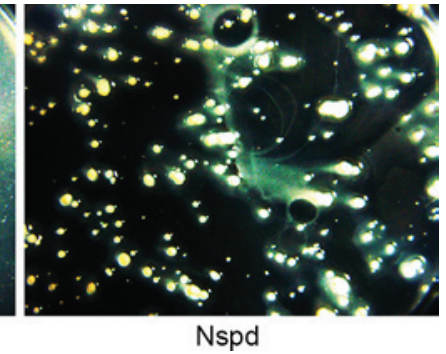

Figure 2. Stereomicroscopic images of Streptococcus mutans biofilms that had formed by $96 \mathrm{~h}$ in the experimental and control groups. The biofilms were observed and images were captured using a stereomicroscope at a magnification of $\mathrm{x} 5$. Nspd, norspermidine.

of the S. mutans pathways regulated by Nspd is presented in a volcano graph (Fig. 8). Volcano graphs demonstrated a large difference in the levels of gene expression in the Nspd group compared with those of the control group (Fig. 8A), and several up or downregulated pathways were observed in the Nspd group (Fig. 8B and C).

$>400$ genes were differentially expressed in the Nspd group compared with the control group (Table I). Since the present study focused on biofilm-associated changes, information induced by Nspd, the present study selected 24 genes that have been fully reported to be closely associated with $S$. mutans biofilm formation and polyamine transport. Among these 24 genes, the expression levels of 12 genes were significantly different $(\mathrm{P}<0.05)$, including $\operatorname{luxS}, \operatorname{com} C, \operatorname{comD}, \operatorname{com} E$, gtfB, $s p a, s c r R, b r p A, f r u B, g b p D$ and $p o t B$. Although the expression levels of the other genes were not significantly different at the 1.5-fold change limit ( $\mathrm{P}>0.05)$, the changes in their expression revealed certain discrete and important tendencies associated 

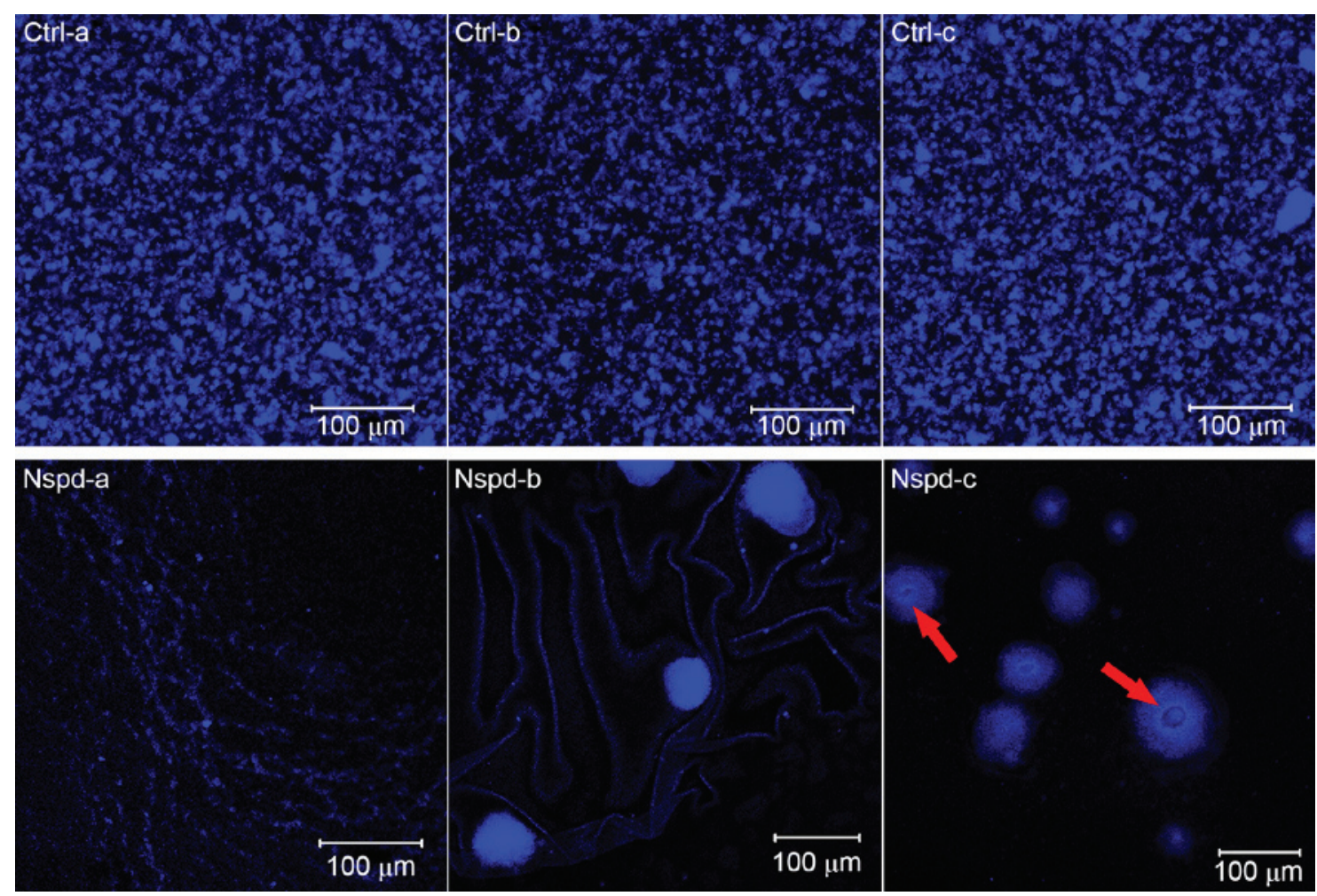

Figure 3. Exopolysaccharide structure of Streptococcus mutans biofilms incubated for $96 \mathrm{~h}$ in the experimental and control groups. Control-a, -b and -c show uniform and regular EPS granules of different areas in the control group. Nspd-a, fishnets EPS structures in the experimental group; Nspd-b, chain-like EPS structures in the experimental group; Nspd-c, star like EPS structures in the experimental group. The red arrows indicate the central hole within a polysaccharide-containing structure. The polysaccharides of the biofilms were stained blue using $300 \mathrm{mg} / \mathrm{l}$ Fluorescent Brightener 28 (magnification x200 or x100; scale bars, $100 \mu \mathrm{m})$. Nspd, norspermidine; Ctrl, control.
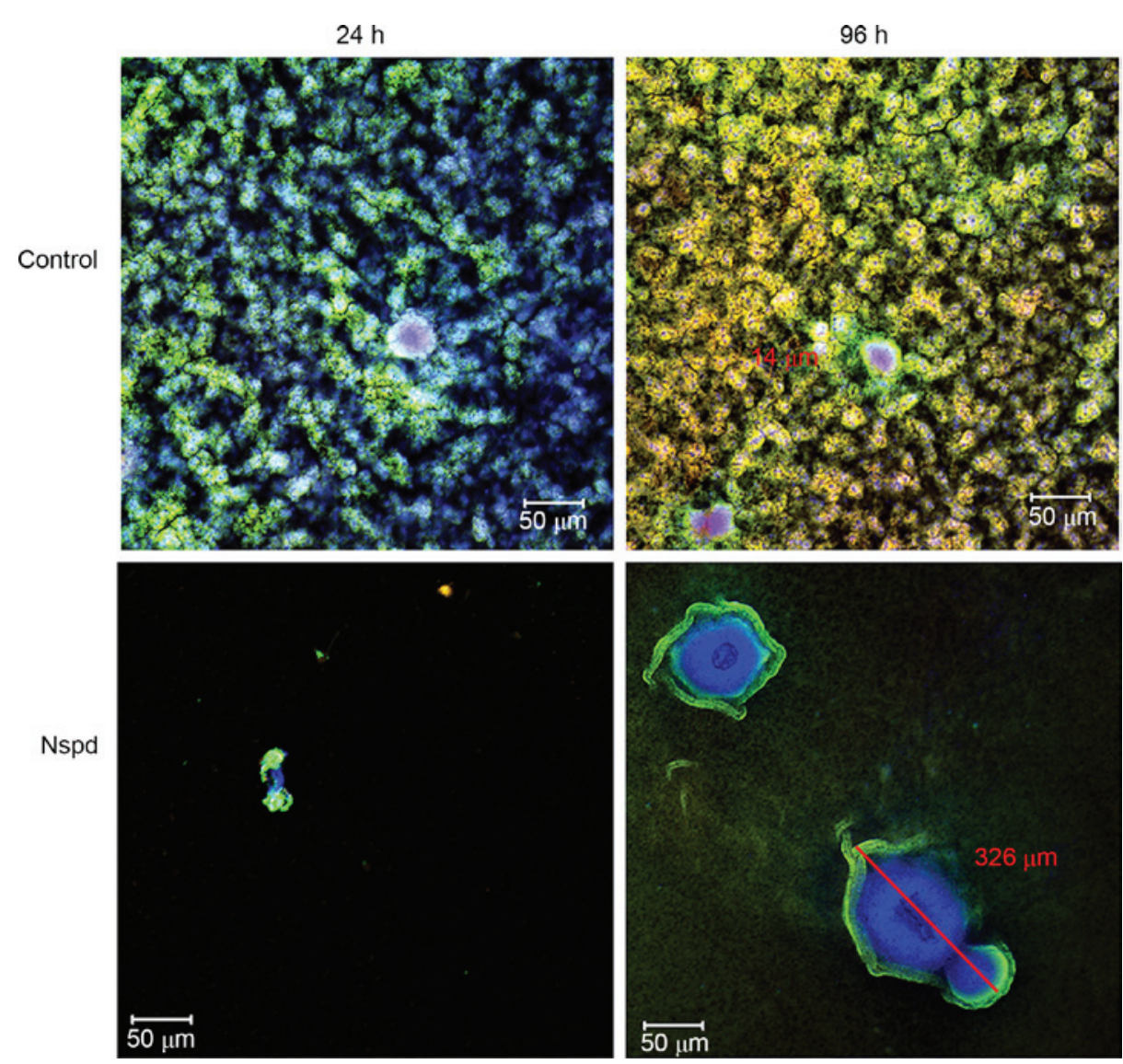

Figure 4. Three-dimensional distribution of exopolysaccharide and Streptococcus mutans cells in the two groups. The biofilms were incubated for 24 and $96 \mathrm{~h}$, and stained using Fluorescent Brightener 28 and L-7012 to label the live (green) and dead (red) cells (scale bar, $50 \mu \mathrm{m}$ ). Nspd, norspermidine. 


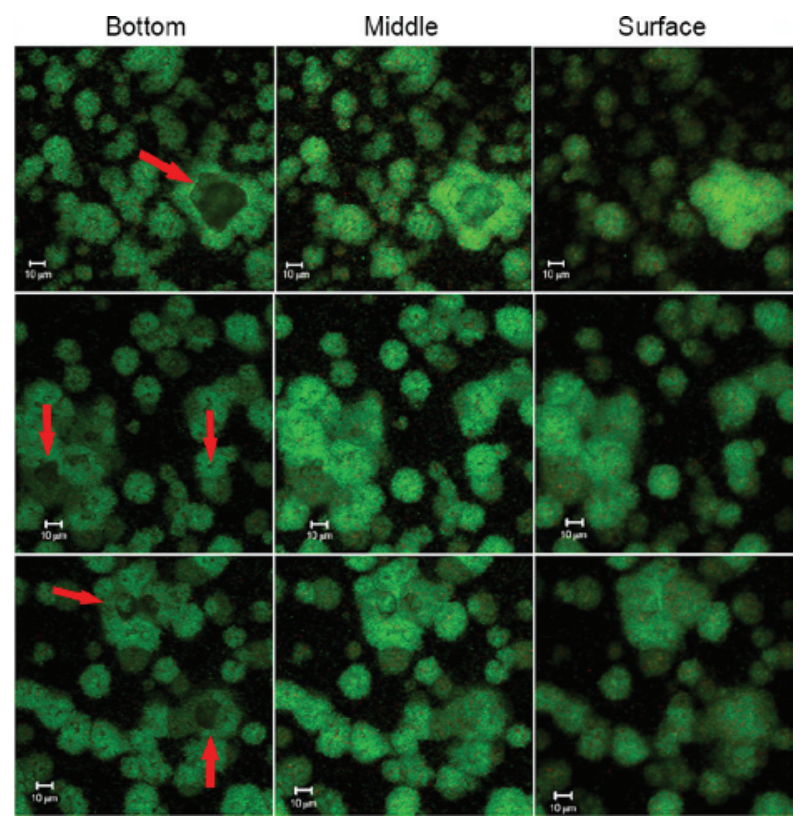

Figure 5. Bacteria-containing 'hollow sphere' structures in the Streptococcus mutans biofilms. The images are sections of 3D imaging demonstrating representative images from the bottom, middle and surface of the biofilms. The biofilms were formed in brain heart infusion broth containing $1 \%$ sucrose during a $12 \mathrm{~h}$ incubation and were stained using the LIVE/DEAD kit to label the live (green) and dead (red) cells. The red arrows indicate a 'space' in a spherical structure (magnification x200; scale bar, $10 \mu \mathrm{m}$ ).

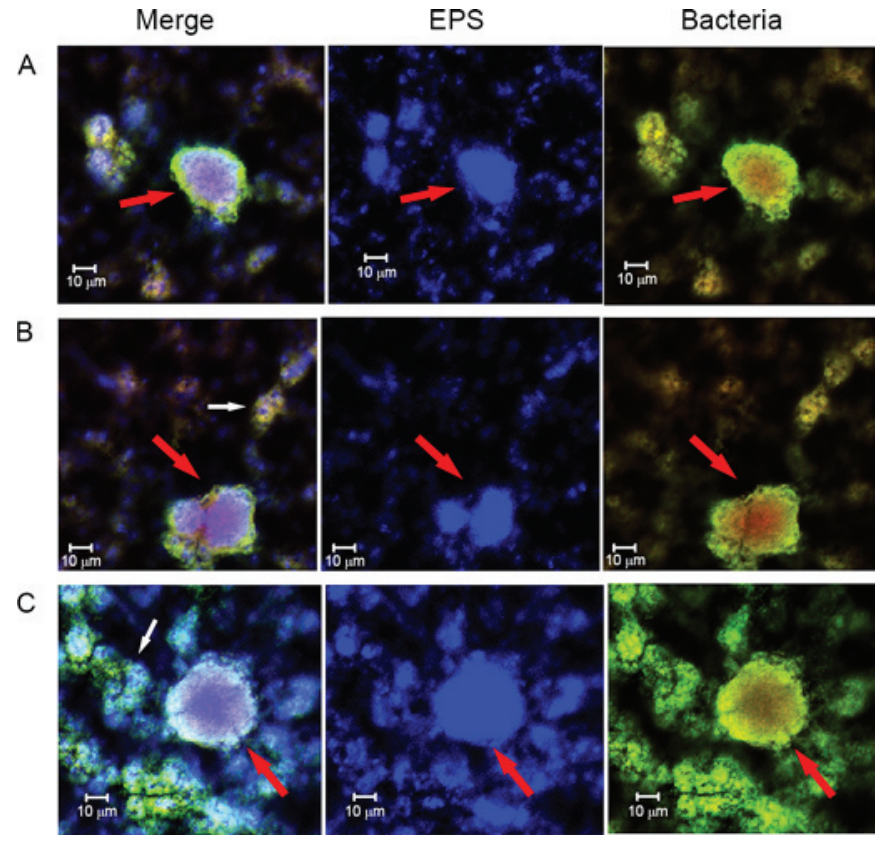

Figure 6. Normal BEUs in Streptococcus mutans biofilms. The images in the Merge row reveal the EPS and bacteria. Images in the EPS row highlight the EPS with the units and the images in the Bacteria row show the bacteria on the exterior of the units. The red arrows indicate typical BEUs consisting of bacteria on the exterior and EPS in the interior. (A) A single BEU with bacteria surrounding an EPS pearl. (B) A combination of two BEUs. The white arrow indicates a typical toroid combined-BEU structure. (C) A single BEU with bacteria surrounding the EPS. The white arrow indicates a rough chain-like combined-BEU structure. The biofilms were stained using $300 \mathrm{mg} / \mathrm{l}$ Fluorescent Brightener 28 to label the polysaccharides (blue), and a L-7012 LIVE/DEAD Viability kit to label the live (green) and dead (red) cells (scale bar, $10 \mu \mathrm{m}$ ). EPS, exopolysaccharide; BEUs, bacteria-EPS units.

with the differences in the biofilm structures in the Nspd group. It was noted that the expression of the QS-related genes lusX,
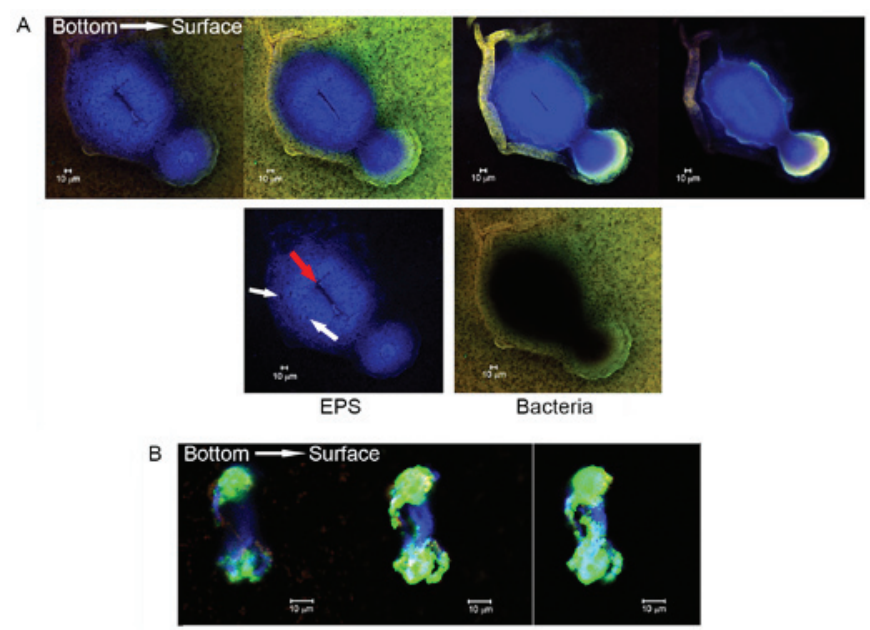

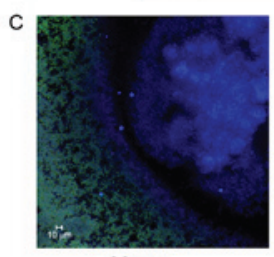

Merge

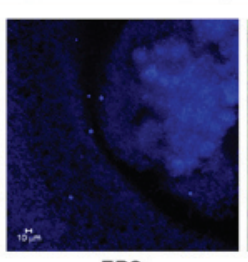

EPS

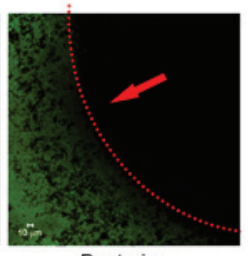

Bacteria
Figure 7. Huge BEUs in the Streptococcus mutans biofilms of the Nspd group. (A) Typical large-scale mature BEUs. The first panel shows optical sections of a unit starting at the adherent interface (bottom) and moving to the upper surface of biofilm surrounded by liquid. The second panel shows detailed views of separate and merged images of the EPS and bacterial cells. (B) Optical sections showing initially formed irregular BEUs, starting at the bottom and moving to the surface. These images show that the bacterial cells secreted EPS toward the center of the units and the bottom interface. (C) A typical spherical interface between the bacteria and EPS of developing BEUs. This image shows the cellular arrangement and the EPS-secretion pattern. The biofilms were stained using $300 \mathrm{mgl}$ Fluorescent Brightener 28 to label the polysaccharides (blue), and a L-7012 LIVE/DEAD Viability kit to label the live (green) and dead (red) cells. The red arrows indicates typical basic units consisting of bacteria on the exterior and EPS in the interior (scale bar, $10 \mu \mathrm{m})$. EPS, exopolysaccharide; BEUs, bacteria-EPS units. 
Table I. Structure of biofilm differentially expressed genes regulated by Nspd relative to the negative control group.

\begin{tabular}{|c|c|c|c|}
\hline Gene name & Predicted function & Regulation & Fold-change \\
\hline gtfA & Sucrose phosphorylase GtfA & Down & 1.5509206 \\
\hline $\operatorname{gtfB}^{\mathrm{a}}$ & Glucosyltransferase-I & Down & 1.4373798 \\
\hline gtfC & Glucosyltransferase-SI & Down & 2.0291426 \\
\hline gtfD & Glucosyltransferase-S & Down & 1.9489628 \\
\hline $\operatorname{scrR}^{\mathrm{a}}$ & Sucrose operon repressor & Up & 1.5087006 \\
\hline $\operatorname{spaP}^{a}$ & Cell surface antigen $\mathrm{SpaP}$ & Down & 19.62776 \\
\hline $\operatorname{brpA}^{\mathrm{a}}$ & Transcriptional regulator & Up & 2.3073087 \\
\hline fruB $^{\mathrm{a}}$ & Exo-beta-D-fructosidase & Down & 1.9644824 \\
\hline relA & Stringent response protein, ppGpp synthetase & Up & 2.0203063 \\
\hline $\operatorname{dex} A$ & Dextranase & Up & 1.6448688 \\
\hline $\operatorname{dexB} B^{a}$ & Dextran glucosidase DexB & Up & 2.9510176 \\
\hline $\operatorname{lux} S^{\mathrm{a}}$ & S-ribosylhomocysteinase & Down & 7.883619 \\
\hline $\operatorname{comC}^{\mathrm{a}}$ & Competence stimulating peptide & Down & 2.6926205 \\
\hline $\operatorname{comD}^{\mathrm{a}}$ & Histidine kinase of the competence regulon, ComD & Down & 7.9834288 \\
\hline $\operatorname{comE}^{\mathrm{a}}$ & Response regulator of the competence regulon ComE & Down & 15.048688 \\
\hline gbpA & Glucan-binding protein GbpA & Up & 1.5979749 \\
\hline gbpB & Secreted antigen $\mathrm{GbpB} / \mathrm{Sag} \mathrm{A}$ & Up & 1.5592909 \\
\hline gbpC & Glucan-binding protein GbpC & Up & 1.2180864 \\
\hline $\operatorname{gbpD}^{\mathrm{a}}$ & Glucan-binding protein D & Up & 3.98988328 \\
\hline potA & Spermidine/putrescine ABC transporter ATP-binding protein & Up & 1.3464806 \\
\hline $\operatorname{potB}^{\mathrm{a}}$ & Spermidine/putrescine $\mathrm{ABC}$ transporter permease & Down & 1.7071223 \\
\hline potC & Spermidine/putrescine ABC transporter permease & Down & 1.1213877 \\
\hline potD & $\mathrm{ABC}$ transporter periplasmic spermidine/putrescine-binding protein & Down & 1.2626697 \\
\hline
\end{tabular}

${ }^{\text {a }}$ Significance was set at a $\mathrm{P}=0.05$.

A

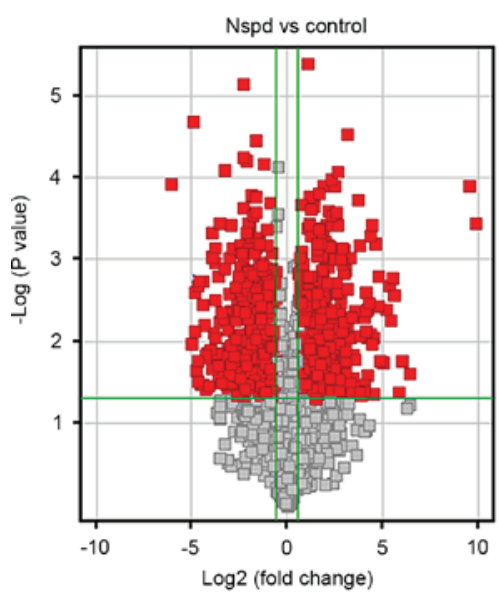

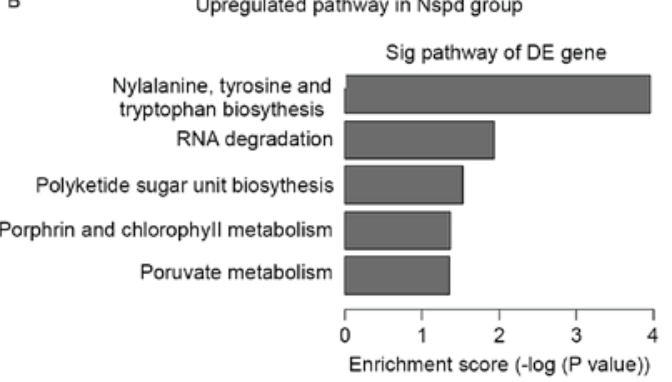

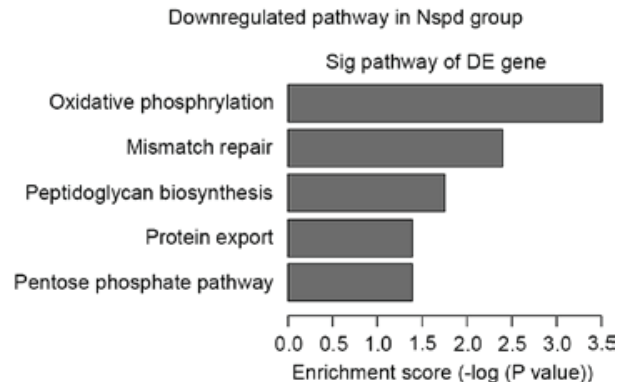

Figure 8. Overview of the Streptococcus mutans genes and pathways regulated by Nspd. (A) Volcano plots to visualize the differential gene expression in the Nspd group compared with the control group. The vertical lines correspond to a 1.5 -fold difference in expression levels, and the horizontal line represents a $\mathrm{P}=0.05$. The red dots in the plot represent mRNAs that were differentially expressed at significant levels. (B) Pathways that were upregulated in the Nspd group compared with the control group. (C) Pathways that were downregulated in the Nspd group compared with the control group. These data were obtained using the latest Kyoto Encyclopedia of Genes and Genomes database. The name of the pathway is noted on the left. The P-value indicates the significance of differential expression of the genes in the pathway. The smaller the $\mathrm{P}$-value, the more significantly differentially expressed were the genes in the pathway $(\mathrm{P}=0.05$ was the cut-off). The P-value was calculated using Fisher's exact test. The enrichment scores were expressed as -log10 units (P-value). Nspd, norspermidine; Sig, signaling; DE, differentially expressed. 


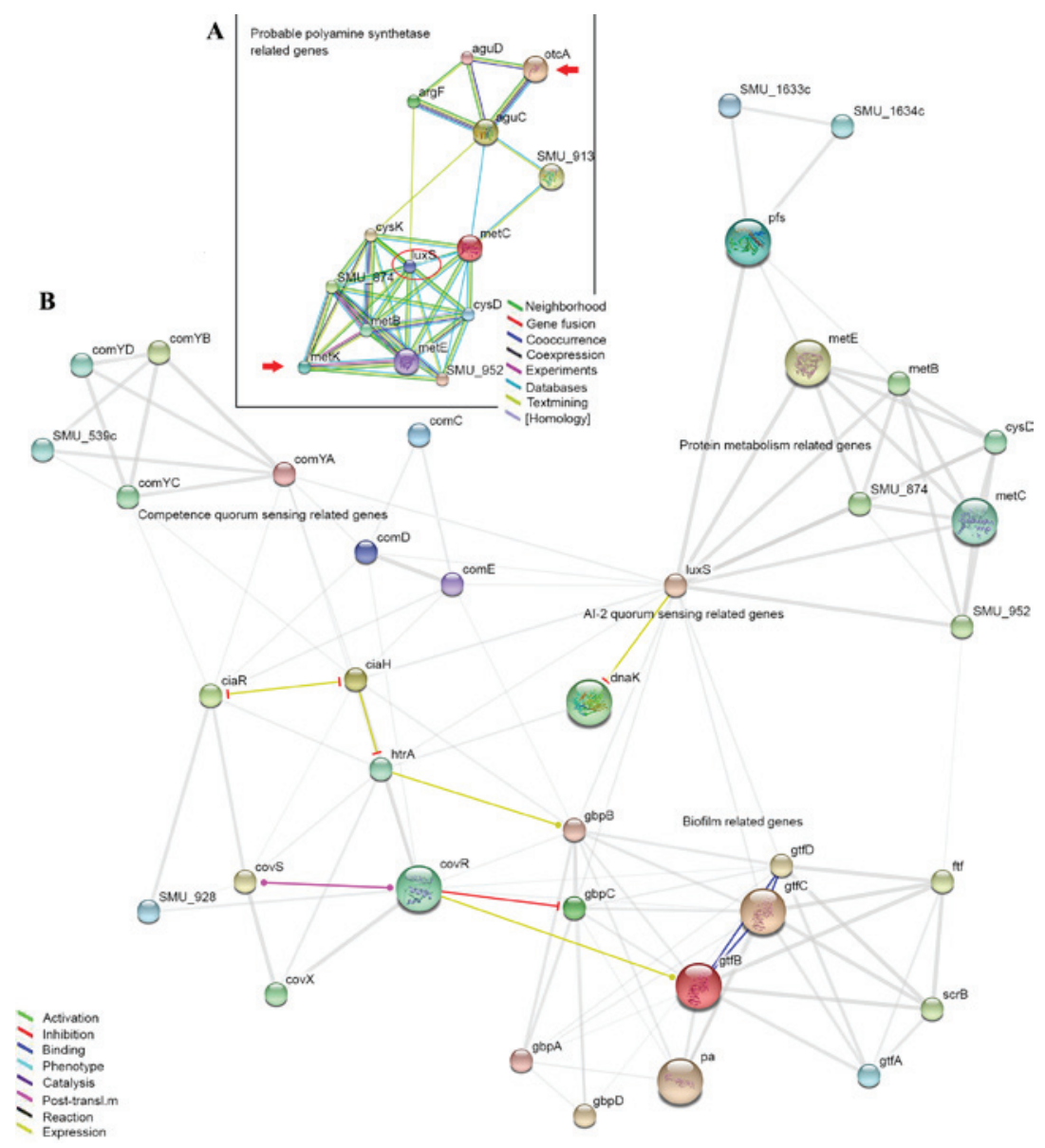

Figure 9. Network map of biofilm-associated genes, QS-associated genes and polyamine-associated genes. (A) The relationship among the polyamine-synthetase genes and the luxS gene. (B) The regulation of the biofilm-associated genes by the competence QS genes and AI-2 QS genes. This figure was created by our research group using the program at http://string-db.org/newstring.

com $C, \operatorname{com} D$ and $c o m E$ were remarkably downregulated by 7.9, 2.7, 8 and 15-fold, respectively $(\mathrm{P}<0.05)$.

Gene connection map. To discover the associations between the genes that were differently expressed led by Nspd and biofilm structure, the present study used an online program that demonstrated the connections among genes (http://string-db.org/newstring.) and created a gene net map (Fig. 9) according to a previous study (21). GTF encoded genes (gtfA, B, C and D) and glucan-binding proteins encoded genes (gbpA, B, C and D) directly affected biofilm architecture and structure. The QS-associated genes included luxS and competence-stimulating peptide $\operatorname{com} C, \operatorname{com} D$ and $\operatorname{com} E$ genes. Glucan production and binding assocaited genes ( $g t f A$, $B, C$ and $D$ and $g b p A, B, C$ and $D$ ) were directly or indirectly impacted by $\operatorname{lux} S, \operatorname{com} C, \operatorname{com} D$ and $\operatorname{com} E$. Certain clues were observed between polyamine and biofilms using this map. otc $A$ encodes putrescine carbamoyltransferase and met $K$ encodes argcarbomyltransferase. This gene-connection map revealed that these polyamine-synthetic genes were most likely associated with gtf and gbp through the luxS gene (Fig. 9B).

\section{Discussion}

Although information associated with the $S$. mutans $\mathrm{Spd} / \mathrm{Put}$ transporter can be observed in NCBI, no report is available concerning these polyamines in S. mutans; the spermidine synthetase remains unknown. Exogenous Nspd, the allosteric form of spermidine, served an adverse role in S. mutans by inhibiting its growth and leading to the formation of irregular biofilm structures. No direct effect of Nspd on S. mutans EPS was observed.

Notably, through observing the different three-dimensional structures of the biofilm that were formed in the presence of Nspd, the present research revealed that the basic structure in $S$. mutans biofilms is composed of EPS surrounded by bacterial units. This clear and distinct unit has not been reported previously and it was termed it as 'bacteria-EPS units' (BEUs; Figs. 6 and 7), as it is more visual in structural detail than colony forming units. This finding may indicate the mechanism by which EPS and bacteria assemble the EPS-rich $S$. mutans biofilms formed in the presence of sucrose and somewhat explain the QS mechanism.

In the present research, spherical or globular BEUs were formed by bacterial clusters secreting EPS into the center of cell clusters. The EPS were surrounded by bacteria rather than the EPS, enmeshing the bacteria in this type of basic structure. This result is in disagreement with certain theories. Xiao et al (7) has reported that EPS were detected surrounding and covering microcolonies, and suggested that individual microcolonies encased in polysaccharides may serve as the architectural units in mixed-species biofilms, 


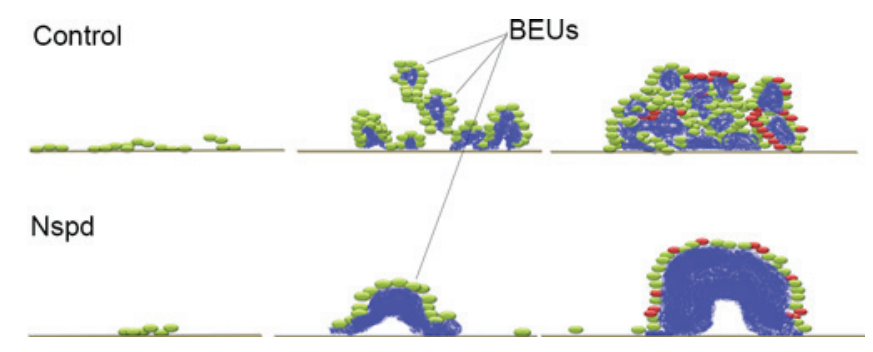

Figure 10. A schematic drawing of the three stages of Streptococcus mutans biofilm formation via BEUs in the control, Spd and Nspd groups. Nspd, norspermidine; BEU, bacteria-exopolysaccharide units.

including those of $S$. mutans (7). These structures were termed EPS-microcolony complexes. As noted in the present study, the diameter of the EPS-microcolony complexes was $>40 \mu \mathrm{m}$ compared with the 5-40 $\mu \mathrm{m}$ diameter of the BEUs observed in the present study; therefore, it was speculated that the BEU may be the most basic structure formed by $S$. mutans. Time-lapse observations of organized BEUs of a larger size that occurred in the Nspd group provided further evidence to support this speculation. This type of organized BEU formed from bacterial clusters that secreted EPS toward the adhesion interface and the center of the cluster, and developed from a hat-like structure into a globular structure. The present study speculated that the bacteria gradually moved toward the exterior, as the EPS granule grew larger. This type of EPS secretion pattern is somewhat similar to the pattern of enamel secretion by ameloblasts (22). However, how the internal EPS protects the bacteria in a basic structure such as a BEU remains to be determined. It was suggested that the development of the biofilm may answer this question; as the biofilm grew, more BEUs formed and they became larger and more densely packed, until they finally contacted one another and the bacteria were crowded within adjacent BEUs (Fig. 10). A biofilm contains a large numbers of BEUs, which would result in the majority of the bacteria being enmeshed within the EPS.

Nspd treatment led to the formation of oversized and undersized BEUs. The present study also proposed that EPS secretion and accumulation at too rapid a rate can lead to some of the defects observed in the oversized BEU-EPS aggregates in the Nspd group. These defects included central holes and some depressions (Fig. 7). It was suggested that the central holes in the oversized BEUs in the Nspd group can be the early gathering sites of the bacteria and the original EPS-secretion points; clear evidence of this hypothesis would be to trace the tracks of the bacteria while they are secreting EPS.

Hydrodynamics, nutrient concentrations, carbon sources, motility, genetics, QS (23-25) and glucan-binding proteins (26) have been identified as factors that affect biofilm architecture. To understand how $S$. mutans regulates or controls the size of the BEUs and forms a regular, smooth, homogeneous biofilm, whole genome expression microarray analysis of $S$. mutans cells in the biofilms formed in the Nspd and control groups was performed with the goal of determining the cause of the differences in biofilm formation. Using the same conditions without agitation, the present study was able to exclude hydrodynamics, nutrient concentration and genetic factors as responsible for the differences between the two groups. Therefore, our attention was focused on alterations in the expression of the cell-surface antigen SpaP, glucosyltransferase, glucan-binding proteins and members of regulatory systems, including the QS system and $\operatorname{cov} R$.

For the Nspd group, the present study focused on 20 genes with severely compromised expression that served a role in biofilms. It was also revealed that the expression of a gene encoding a surface antigen I (SpaP), which is important for the initial attachment stage of biofilm formation (27-31), was markedly decreased. This phenomenon can explain why most areas consisted of a rather thin layer of bacteria or contained no bacteria. A sharp reduction in the ability of bacteria to adhere to the surface led to dramatic deficiencies in the biofilm basement. EPS are an important component of the microbial biofilm extracellular matrix as it contributes to the overall biofilm architecture and to the resistance phenotype of the bacteria in biofilms (5-33). An irregular architecture results in the loss of protection of the bacteria and leads to decreased virulence. In $S$. mutans biofilms, the EPS are composed predominantly of glucans. The glucosyltransferases (Gtfs) of S. mutans are constituents of the pellicle and can synthesize glucans in situ using sucrose $(34,35)$. The polymers that form on the surface provide bacterial binding sites for subsequent colonization and local accumulation of $S$. mutans and other organisms $(36,37)$. Gtfs also bind numerous oral bacteria, even those that do not synthesize Gtfs $(8,35,38)$, thereby converting them into original glucan producers (35). In the Nspd group, the expression levels of four glucosyltransferase-encoding genes, gtfA, $B, C$ and $D$ (39-42) were all reduced compared with the levels of the control group. Although a number of oversized EPS structures formed in the biofilms of the Nspd group, the total quantity of EPS was less than that of the control group. The glucan-binding protein family includes the glucan-protein receptor, the function of which is necessary to sustain the architecture of biofilms (26,43-45). The effect of such proteins was demonstrated by the large structures containing EPS in the Nspd group. The expression levels of four glucan-binding protein-encoding genes, $g b p A, B, C$ and $D$, were all upregulated in the Nspd group. The overexpression of these genes may have led to too much EPS binding and induced the formation of the huge aggregates of EPS mentioned above.

SpaP, glucosyltransferase and glucan-binding proteins are considered direct factors that affect the architecture of biofilms, whereas the QS system indirectly regulates biofilm architecture by affecting the above-mentioned proteins. In the present study, the expression levels of $\operatorname{com} C, \operatorname{com} D, \operatorname{com} E$ and lux $S$ were markedly reduced in the Nspd group. As mentioned in above, $S$. mutans produces both AI-2 and competence-stimulating peptides (CSP belongs to the AI-1 group) via the luxS and $\operatorname{com} C$ genes, respectively. The relationship between QS and $S$. mutans biofilm structure has been studied and discussed by a certain number of researchers $(26,46,47)$. Inactivation of any of the individual genes under investigation results in the formation of an abnormal biofilm. The $\operatorname{com} C$ deletion mutant, which is unable to produce or secrete CSP, forms biofilms with an altered architecture, whereas the comD and comE $S$. mutans mutants, which are defective in sensing and responding to CSP, form biofilms with a reduced biomass (46). Previous reports regarding the effect of an interspecies QS system on the architecture of $S$. mutans biofilms have attracted our attention. Biofilms formed by the luxS mutant have a more 
granular appearance compared with the relatively smooth, confluent layer normally produced by wild-type cells $(47,48)$. This granular appearance was similar to the appearance of the biofilm formed by the Nspd group in the present study. Huang et al (49) reported that biofilm formation by the luxS mutant was accelerated during the mid-exponential phase and that the differences between the mutant and wild-type biofilms were markedly greater when carbohydrates were added to the medium. These findings suggested that AI-2 may affect the early stages of biofilm formation by inhibiting exopolysaccharide matrix production (49). Consistent with these previous reports (47-49), luxS expression was notably downregulated in the Nspd group in the present study, which may have led to the acceleration of EPS aggregation, and subsequently led to the formation of oversized BEUs.

In conclusion, the present study proposed a mechanism for the assembly of three-dimensional structures in S. mutans biofilms. The limited size and regularity of the BEUs, due to regulation by the QS system, guaranteed that the majority of the bacteria dispersed themselves among and covered the EPS, which protected the $S$. mutans bacteria in the biofilm from unfavorable factors. The following appeared to explain certain aspects of the formation of normal BEUs: i) The size of the EPS granule in the BEU was predetermined and limited; ii) The bacteria were crowded by more than surrounded by the EPS in the biofilm; iii) The bacteria secreted EPS into the center of the aggregates while moving toward the exterior; iv) The interface between the bacteria and EPS was a globular interface; and v) The BEUs always combined to form clusters.

\section{Acknowledgements}

The authors would like to thank Professor Christine Wu and Dr Wei Li (College of Dentistry, University of Illinois) for their assistance and advice in repeating the experiments. Professor Yutao Jian, Mr. Jingtao Wang and Mr. Tao He (Provincial Key Laboratory of Stomatology, Sun Yat-sen University) for their assistance in the confocal laser-scanning microscopic analysis. The present study was supported by the National Natural Science Foundation of China (no. 81371132). The funders had no role in the study design, data collection and analysis, decision to publish, or preparation of the manuscript.

\section{References}

1. Wallace HM, Fraser AV and Hughes A: A perspective of polyamine metabolism. Biochem J 376: 1-14, 2003.

2. Romero D and Kolter R: Will biofilm disassembly agents make it to market? Trends Microbiol 19: 304-306, 2011.

3. Flemming HC, Neu TR and Wozniak DJ: The EPS matrix: The 'house of biofilm cells'. J Bacteriol 189: 7945-7947, 2007.

4. Flemming $\mathrm{HC}$ and Wingender J: The biofilm matrix. Nat Rev Microbiol 8: 623-633, 2010.

5. Branda SS, Vik S, Friedman L and Kolter R: Biofilms: The matrix revisited. Trends Microbiol 13: 20-26, 2005.

6. Bowen WH and Koo H: Biology of Streptococcus mutans-derived glucosyltransferases: Role in extracellular matrix formation of cariogenic biofilms. Caries Res 45: 69-86, 2011.

7. Xiao J, Klein MI, Falsetta ML, Lu B, Delahunty CM, Yates JR III, Heydorn $\mathrm{A}$ and Koo $\mathrm{H}$ : The exopolysaccharide matrix modulates the interaction between 3D architecture and virulence of a mixed-species oral biofilm. PLoS Pathog 8: e1002623, 2012.

8. Hamada S, Tai S and Slade HD: Binding of glucosyltransferase and glucan synthesis by Streptococcus mutans and other bacteria. Infect Immun 21: 213-220, 1978.
9. Koo H, Xiao J, Klein MI and Jeon JG: Exopolysaccharides produced by Streptococcus mutans glucosyltransferases modulate the establishment of microcolonies within multispecies biofilms. J Bacteriol 192: 3024-3032, 2010.

10. Loesche WJ: Role of Streptococcus mutans in human dental decay. Microbiol Rev 50: 353-380, 1986.

11. Fuqua C, Parsek MR and Greenberg EP: Regulation of gene expression by cell-to-cell communication: Acyl-homoserine lactone quorum sensing. Annu Rev Genet 35: 439-468, 2001.

12. Kleerebezem M and Quadri LE: Peptide pheromone-dependent regulation of antimicrobial peptide production in Gram-positive bacteria: A case of multicellular behavior. Peptides 22: 1579-1596, 2001.

13. Miller MB and Bassler BL: Quorum sensing in bacteria. Annu Rev Microbiol 55: 165-199, 2001

14. Miller MB, Skorupski K, Lenz DH, Taylor RK and Bassler BL: Parallel quorum sensing systems converge to regulate virulence in Vibrio cholerae. Cell 110: 303-314, 2002.

15. Shapiro JA: Thinking about bacterial populations as multicellular organisms. Annu Rev Microbiol 52: 81-104, 1998.

16. Karatan E and Watnick P: Signals, regulatory networks, and materials that build and break bacterial biofilms. Microbiol Mol Biol Rev 73: 310-347, 2009.

17. Wortham BW, Oliveira MA, Fetherston JD and Perry RD: Polyamines are required for the expression of key Hms proteins important for Yersinia pestis biofilm formation. Environ Microbiol 12: 2034-2047, 2010.

18. Sakamoto A, Terui Y, Yamamoto T, Kasahara T, Nakamura M, Tomitori H, Yamamoto K, Ishihama A, Michael AJ, Igarashi K and Kashiwagi K: Enhanced biofilm formation and/or cell viability by polyamines through stimulation of response regulators UvrY and CpxR in the two-component signal transducing systems and ribosome recycling factor. Int J Biochem Cell Biol 44: 1877-1886, 2012.

19. Sturgill G and Rather PN: Evidence that putrescine acts as an extracellular signal required for swarming in Proteus mirabilis. Mol Microbiol 51: 437-446, 2004.

20. Kolodkin-Gal I, Cao S, Chai L, Böttcher T, Kolter R, Clardy J and Losick R: A self-produced trigger for biofilm disassembly that targets exopolysaccharide. Cell 149: 684-692, 2012.

21. Szklarczyk D, Franceschini A, Wyder S, Forslund K, Heller D, Huerta-Cepas J, Simonovic M, Roth A, Santos A, Tsafou KP, et al: STRING v10: Protein-protein interaction networks, integrated over the tree of life. Nucleic Acids Res 43 (Database Issue): D447-D452, 2015.

22. Nishikawa S: Correlation of the arrangement pattern of enamel rods and secretory ameloblasts in pig and monkey teeth: A possible role of the terminal webs in ameloblast movement during secretion. Anat Rec 232: 466-478, 1992.

23. Harmsen M, Yang L, Pamp SJ and Tolker-Nielsen T: An update on Pseudomonas aeruginosa biofilm formation, tolerance and dispersal. FEMS Immunol Med Microbiol 59: 253-268, 2010.

24. Stoodley P, Dodds I, Boyle JD and Lappin-Scott HM: Influence of hydrodynamics and nutrients on biofilm structure. J Appl Microbiol 85 (Suppl 1): 19S-28S, 1998.

25. Parsek MR and Tolker-Nielsen T: Pattern formation in Pseudomonas aeruginosa biofilms. Curr Opin Microbiol 11: 560-566, 2008.

26. Lynch DJ, Fountain TL, Mazurkiewicz JE and Banas JA: Glucan-binding proteins are essential for shaping Streptococcus mutans biofilm architecture. FEMS Microbiol Lett 268: 158-165, 2007.

27. Crowley PJ, Brady LJ, Michalek SM and Bleiweis AS: Virulence of a spaP mutant of Streptococcus mutans in a gnotobiotic rat model. Infect Immun 67: 1201-1206, 1999.

28. Qu YP, Liu JG, Yang P and Yang DQ: Construction and expression of spap/A eukaryotic expression plasmid of Streptococcus mutans in mammalian cells. Shanghai Kou Qiang Yi Xue 18: 61-65. 2009 (In Chinese).

29. Durán-Contreras GL, Torre-Martínez HH, de la Rosa EI, Hernández RM and de la Garza Ramos M: spaP gene of Streptococcus mutans in dental plaque and its relationship with early childhood caries. Eur J Paediatr Dent 12: 220-224, 2011.

30. Davis E, Kennedy D, Halperin SA and Lee SF: Role of the cell wall microenvironment in expression of a heterologous SpaP-S1 fusion protein by Streptococcus gordonii. Appl Environ Microbiol 77: 1660-1666, 2011.

31. Sato Y, Okamoto-Shibayama K and Azuma T: A mechanism for extremely weak SpaP-expression in Streptococcus mutans strain Z1. J Oral Microbiol 3, 2011. 
32. Itoh Y, Wang X, Hinnebusch BJ, Preston JR and Romeo T: Depolymerization of beta-1,6-N-acetyl-D-glucosamine disrupts the integrity of diverse bacterial biofilms. J Bacteriol 187: 382-387, 2005

33. Sutherland IW: The biofilm matrix-an immobilized but dynamic microbial environment. Trends Microbiol 9: 222-227, 2001.

34. Rolla G, Ciardi JE and Schultz SA: Adsorption of glucosyltransferase to saliva coated hydroxyapatite. Possible mechanism for sucrose dependent bacterial colonization of teeth. Scand J Dent Res 91: 112-117, 1983.

35. Vacca-Smith AM and Bowen WH: Binding properties of streptococcal glucosyltransferases for hydroxyapatite, saliva-coated hydroxyapatite, and bacterial surfaces. Arch Oral Biol 43: 103-110, 1998

36. Schilling KM and Bowen WH: Glucans synthesized in situ in experimental salivary pellicle function as specific binding sites for Streptococcus mutans. Infect Immun 60: 284-295, 1992.

37. Venkitaraman AR, Vacca-Smith AM, Kopec LK and Bowen WH: Characterization of glucosyltransferaseB, GtfC, and GtfD in solution and on the surface of hydroxyapatite. J Dent Res 74: 1695-1701, 1995

38. McCabe RM and Donkersloot JA: Adherence of Veillonella species mediated by extracellular glucosyltransferase from Streptococcus salivarius. Infect Immun 18: 726-734, 1977.

39. Yamashita Y, Bowen WH and Kuramitsu HK: Molecular analysis of a Streptococcus mutans strain exhibiting polymorphism in the tandem gtfB and gtfC genes. Infect Immun 60: 1618-1624, 1992.

40. Chia JS, Hsieh CC, Yang CS and Chen JY: Purification of glucosyltransferases (GtfB/C and GtfD) from mutant strains of Streptococcus mutans. Zhonghua Min Guo Wei Sheng Wu Ji Mian Yi Xue Za Zhi 28: 1-12, 1995.

41. Tsai YW, Chia JS, Shiau YY, Chou HC, Liaw YC and Lou KL: Three-dimensional modelling of the catalytic domain of Streptococcus mutans glucosyltransferase GtfB. FEMS Microbiol Lett 188: 75-79, 2000.
42. Yoshida A and Kuramitsu HK: Streptococcus mutans biofilm formation: Utilization of a gtfB promoter-green fluorescent protein (PgtfB::gfp) construct to monitor development. Microbiology 148: 3385-3394, 2002.

43. Lynch DJ, Michalek SM, Zhu M, Drake D, Qian F and Banas JA: Cariogenicity of Streptococcus mutans glucan-binding protein deletion mutants. Oral Health Dent Manag 12: 191-199, 2013.

44. Banas JA, Fountain TL, Mazurkiewicz JE, Sun K and Vickerman MM: Streptococcus mutans glucan-binding protein-A affects Streptococcus gordonii biofilm architecture. FEMS Microbiol Lett 267: 80-88, 2007.

45. Banas JA, Hazlett KR and Mazurkiewicz JE: An in vitro model for studying the contributions of the Streptococcus mutans glucan-binding protein A to biofilm structure. Methods Enzymol 337: 425-433, 2001.

46. Li YH, Tang N, Aspiras MB, Lau PC, Lee JH, Ellen RP and Cvitkovitch DG: A quorum-sensing signaling system essential for genetic competence in Streptococcus mutans is involved in biofilm formation. J Bacteriol 184: 2699-2708, 2002.

47. Merritt J, Qi F, Goodman SD, Anderson MH and Shi W: Mutation of luxS affects biofilm formation in Streptococcus mutans. Infect Immun 71: 1972-1979, 2003.

48. Yoshida A, Ansai T, Takehara T and Kuramitsu HK: LuxS-based signaling affects Streptococcus mutans biofilm formation. Appl Environ Microbiol 71: 2372-2380, 2005.

49. Huang Z, Meric G, Liu Z, Ma R, Tang Z and Lejeune P: luxS-based quorum-sensing signaling affects Biofilm formation in Streptococcus mutans. J Mol Microbiol Biotechnol 17: 12-19, 2009. 